\title{
Normal and inverse current-induced magnetization switching in a single nanopillar
}

H. Dassow, R. Lehndorff, D. E. Bürgler, M. Buchmeier, P. A. Grünberg, C. M. Schneider, and A. van der Hart

Citation: Appl. Phys. Lett. 89, 222511 (2006);

View online: https://doi.org/10.1063/1.2398923

View Table of Contents: http://aip.scitation.org/toc/apl/89/22

Published by the American Institute of Physics

\section{Articles you may be interested in}

Magnetic surface domain imaging of uncapped epitaxial FeRh(001) thin films across the temperature-induced metamagnetic transition

AIP Advances 6, 015211 (2016); 10.1063/1.4940758

Interface-mediated ferroelectric patterning and $\mathrm{Mn}$ valency in nano-structured $\mathrm{PbTiO}_{3} / \mathrm{La}_{0.7} \mathrm{Sr}_{0.3} \mathrm{MnO}_{3}$

Journal of Applied Physics 120, 095304 (2016); 10.1063/1.4962007

Full field electron spectromicroscopy applied to ferroelectric materials

Journal of Applied Physics 113, 187217 (2013); 10.1063/1.4801968

Permalloy and $\mathrm{C}_{50} \mathrm{Pd}_{50}$ as ferromagnetic contacts for magnetoresistance measurements in carbon nanotubebased transport structures

Journal of Applied Physics 111, 07B309 (2012); 10.1063/1.3673841

Observation of spin filtering in magnetic insulator contacts to silicon

Applied Physics Letters 98, 142503 (2011); 10.1063/1.3572016

Spin-dependent transport in multiwalled carbon nanotubes

Journal of Applied Physics 91, 7026 (2002); 10.1063/1.1452227

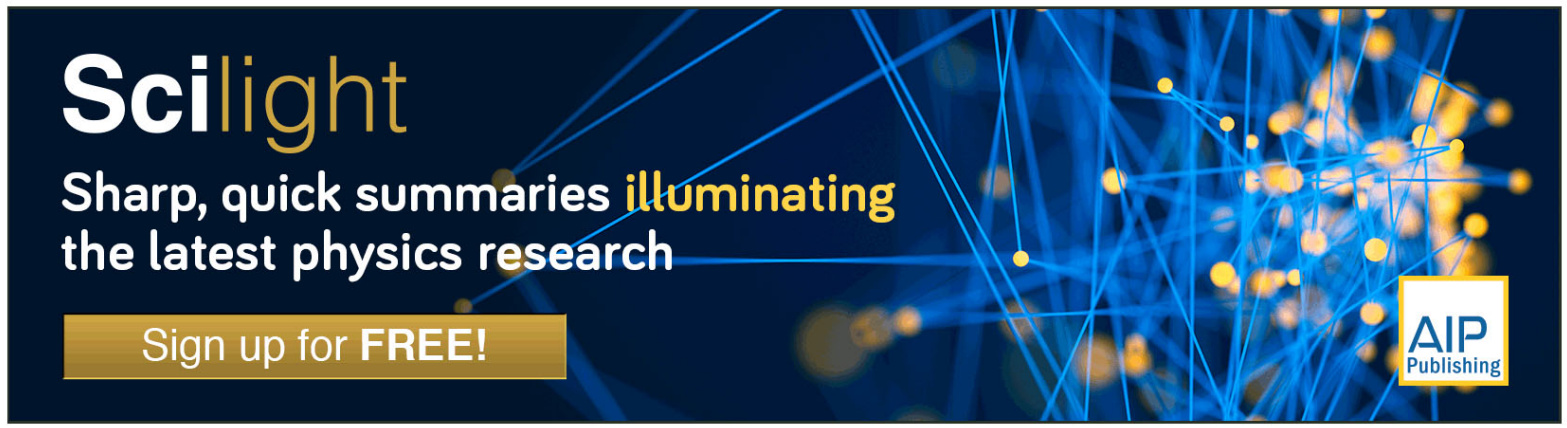




\title{
Normal and inverse current-induced magnetization switching in a single nanopillar
}

\author{
H. Dassow, R. Lehndorff, D. E. Bürgler, ${ }^{\text {a) }}$ M. Buchmeier, \\ P. A. Grünberg, and C. M. Schneider \\ Institute of Solid State Research-Electronic Properties (IFF-9) and cni-Center of Nanoelectronic Systems for \\ Information Technology, Research Center Jülich GmbH, D-52425 Jülich, Germany
}

\begin{abstract}
A. van der Hart
Institute of Thin Films and Interfaces-Process Technology (ISG-PT) and cni-Center of Nanoelectronic Systems for Information Technology, Research Center Jülich GmbH, D-52425 Jülich, Germany
\end{abstract}

(Received 24 March 2006; accepted 18 October 2006; published online 1 December 2006)

\begin{abstract}
The authors report on current-induced magnetization switching (CIMS) in single-crystalline nanopillars. $\mathrm{Fe}(14 \mathrm{~nm}) / \mathrm{Cr}(0.9 \mathrm{~nm}) / \mathrm{Fe}(10 \mathrm{~nm}) / \operatorname{Ag}(6 \mathrm{~nm}) / \mathrm{Fe}(2 \mathrm{~nm})$ multilayers are deposited by molecular-beam epitaxy. The central Fe layer is coupled to the thick one by interlayer exchange coupling over $\mathrm{Cr}$, while the topmost Fe layer is decoupled. Nanopillars with $150 \mathrm{~nm}$ diameter are prepared by optical and e-beam lithographies. The opposite spin scattering asymmetries of the $\mathrm{Fe} / \mathrm{Cr}$ and $\mathrm{Fe} / \mathrm{Ag}$ interfaces enabled the authors to observe normal and inverse CIMS for the two subsystems, which are combined in a single device. At high magnetic fields, steplike resistance changes are measured at positive currents and are attributed to current-driven magnetic excitations. (C) 2006 American Institute of Physics. [DOI: 10.1063/1.2398923]
\end{abstract}

In a magnetic multilayer containing two ferromagnetic (FM) layers and a nonmagnetic (NM) spacer (FM2/NM/ FM1), an electric current flowing perpendicularly to the layers current perpendicular (to the) plane (CPP) gets spin polarized by the FM layers, leading to a giant magnetoresistance (GMR). ${ }^{1,2}$ Thus, spin currents can sense the magnetization state of the magnetic system. Slonczewski ${ }^{3}$ and Berger ${ }^{4}$ first predicted that spin currents of appropriate strength can also directly influence the magnetizations without applying an external magnetic field. Electrons flowing from FM2 to FM1 are first polarized by FM2 and then repolarized at the NM/FM1 interface, where the transverse component of the spin current is absorbed and acts as a torque on the magnetic moment $\boldsymbol{M}_{\mathbf{1}}$ of FM1. ${ }^{5}$ By reversing the current direction, the spin current reflected from FM2 is repolarized at the NM/FM1 interface leading to a reversed torque. Therefore, $\boldsymbol{M}_{\mathbf{1}}$ can be switched from the parallel (P) to the antiparallel (AP) configuration with respect to $\boldsymbol{M}_{\mathbf{2}}$ back and forth by repeatedly reversing the current polarity, as long as $\boldsymbol{M}_{\mathbf{2}}$ remains fixed. This pinning can be achieved by different FM layer thicknesses, ${ }^{6-8}$ by the exchange bias effect, ${ }^{9}$ or by making use of interlayer exchange coupling, as in our case.

In order to achieve large spin-torque effects a high spin polarization $P$ of the current is needed. The present work is motivated by publications of Stiles and Penn ${ }^{10}$ and Stiles and Zangwill, ${ }^{5}$ in which the authors predict high positive spin polarization for single-crystalline $\mathrm{Fe} / \mathrm{Ag}(001)$ interfaces and negative spin polarization for $\mathrm{Fe} / \mathrm{Cr}(001)$ interfaces.

We grow the magnetic multilayer in a molecular-beam epitaxy (MBE) system onto in situ cleaned GaAs(001) substrates $\left(10 \times 10 \mathrm{~mm}^{2}\right)$. We deposit $1 \mathrm{~nm} \mathrm{Fe}$ and $150 \mathrm{~nm} \mathrm{Ag}$ at $100{ }^{\circ} \mathrm{C}$ to get a flat buffer system after annealing at $300{ }^{\circ} \mathrm{C}$ for $1 \mathrm{~h} .{ }^{11}$ The Ag buffer also acts as a bottom elec-

\footnotetext{
a) Author to whom correspondence should be addressed;
} Fax: 49-2461-614-443; electronic mail: d.buergler@fz-juelich.de trode for the transport measurements. The following layers are then deposited at room temperature (RT): $\mathrm{Fe}(14) / \mathrm{Cr}(0.9) /$ $\mathrm{Fe}(10) / \mathrm{Ag}(6) / \mathrm{Fe}(2)$ (thicknesses in nanometers). We check the crystalline surface structure of each layer by low-energy electron diffraction. The spots characteristic of (001) surfaces slightly broaden with increasing total thickness but still indicate high crystalline quality, even for the final $50 \mathrm{~nm}$ $\mathrm{Au}(001)$ capping layer. The bottom and central FM layers $[\mathrm{Fe}(14)$ and $\mathrm{Fe}(10)]$ are coupled by interlayer exchange coupling over the $\mathrm{Cr}$ interlayer.

In Fig. 1 we present a longitudinal magneto-optical Kerr effect (MOKE) hysteresis loop with the magnetic field parallel to an easy [100] axis of the Fe layers in the film plane. The saturation field is $\left|B_{S}\right|=76 \mathrm{mT}$. For smaller magnetic fields the central $\mathrm{Fe}(10)$ layer remagnetizes via a canted state to the fully AP configuration of the stack, which is adopted below $\pm 35 \mathrm{mT}$. In this state, the magnetization of the central $\mathrm{Fe}(10)$ layer points opposite to the field direction because the interlayer coupling dominates over the Zeeman energy. After reversing the field direction we measure another jump, which corresponds to the reversal of the $\mathrm{Fe}(2)$ layer at $\pm 0.3 \mathrm{mT}$. At $\pm 3 \mathrm{mT}$ the two coupled Fe layers reverse simultaneously due

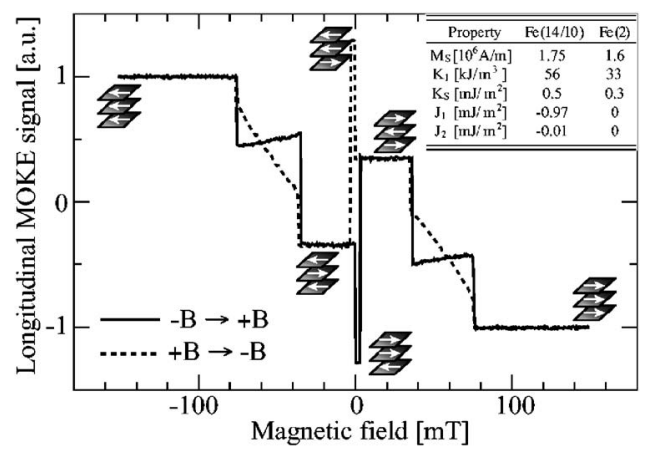

FIG. 1. MOKE hysteresis loop of the extended layered system measured with the field along an easy axis of the Fe layers. 


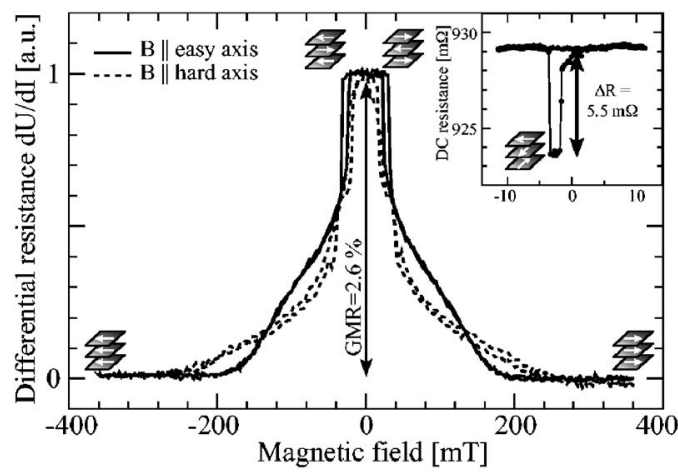

FIG. 2. GMR hysteresis loop with field parallel to an easy (solid) and a hard (dashed) axis. Inset: Minor GMR loop.

to their unequal thicknesses. By fitting the MOKE measurements ${ }^{12}$ and additional Brillouin light scattering measurements ${ }^{13}$ we extract the magnetic properties of each layer as compiled in the inset of Fig. 1. The saturation magnetization $M_{S}$ and the crystalline anisotropy $K_{1}$ have bulk values $^{14,15}$ and indicate the high quality of the layers. Only the thin $\mathrm{Fe}(2)$ layer has reduced $M_{S}$ and $K_{1}$. The negligible coupling constants $J_{1}$ and $J_{2}$ of $\mathrm{Fe}(2)$ show that this layer is decoupled. $K_{S}$ denotes the interface anisotropy.

We have developed a combined optical and e-beam lithography process for the fabrication of nanopillars. First, we define the leads and contact pads of the bottom electrode by using AZ5206 photoresist and ion beam etching (IBE). We then employ hydrogen silsesquioxane (HSQ) as a negative e-beam sensitive resist ${ }^{16}$ and a Leica EBPG 5HR e-beam writer to define small nanopillars. The resist structures are circular and transferred into the magnetic layers by IBE. The timed etching process is stopped inside the magnetic multilayer. Typical diameters of the developed resist structures are 100-150 nm (measured with an atomic force microscope). Due to redeposition during IBE, ${ }^{17}$ the nanopillars broaden to $150-200 \mathrm{~nm}$. The pillars are planarized by spin coating HSQ. Subsequent e-beam exposure turns HSQ into $\mathrm{SiO}_{x}$, which electrically insulates the pillars. ${ }^{16}$ In order to improve the insulation, especially at the sidewalls of the bottom electrodes, a $50 \mathrm{~nm} \mathrm{Si}_{3} \mathrm{~N}_{4}$ layer is deposited by plasma enhanced chemical vapor deposition. We open the top of the nanopillars by IBE and use an optical lift-off process of $300 \mathrm{~nm} \mathrm{Au}$ for the preparation of the top electrode for the four-point resistance measurements.

The dc voltage drop of a constant current $I$ applied to the junction is measured, and by dividing by $I$ we calculate the absolute resistance $R$. The differential resistance $d U / d I$ is recorded with lock-in technique by mixing $I$ with a small modulated voltage $(300 \mu \mathrm{V}, 12 \mathrm{kHz})$. Typical junction resistances lie between 1 and $3 \Omega$. All data presented here are taken at RT.

CPP-GMR loops of a junction at zero dc bias are shown in Fig. 2. The solid (dashed) loop represents the data with magnetic field along an easy (hard) axis of $\mathrm{Fe}(001)$. The curves show a different behavior: The nanopillar exhibits fourfold magnetocrystalline anisotropy and, thus, is still single crystalline. The low field loop in the inset of Fig. 2 shows the absolute resistance measured with a small dc of $1 \mathrm{~mA}$. The drop on the first half of the cycle corresponding to a change of the magnetization direction of $\mathrm{Fe}(2)$ does not occur in every measurement. Thus, the patterning has modi-

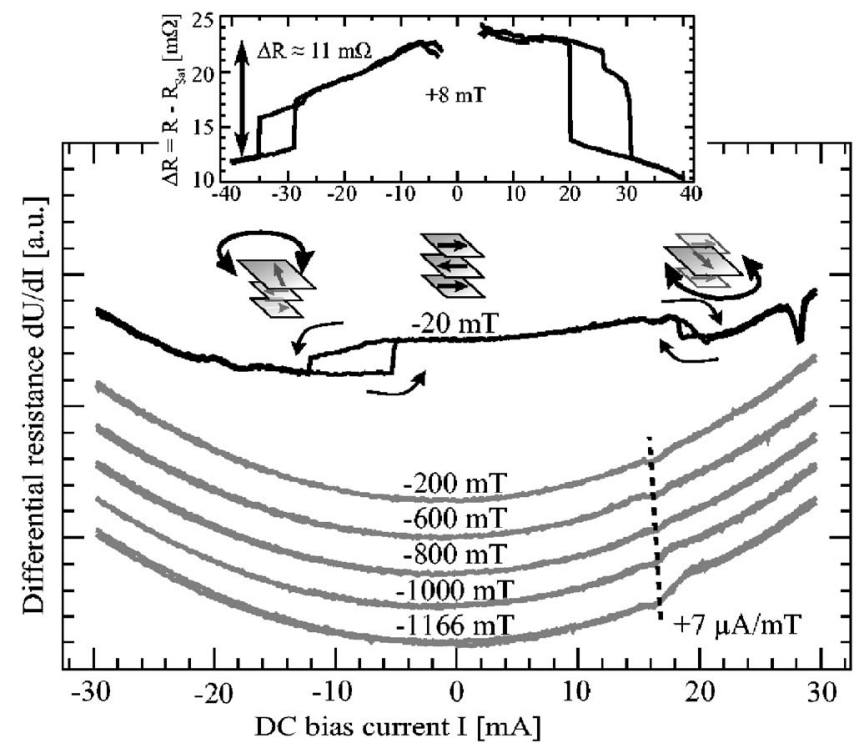

FIG. 3. $(d U / d I)-I$ loops with field parallel to an easy axis. Curves measured at different fields are vertically offset for clarity.

fied the magnetic configuration and the structured $\mathrm{Fe}(2)$ nanomagnet is now coupled to the rest of the system by dipolar stray fields at the edges or by domain wall coupling. This is a common feature in these devices and is also seen in Co nanopillars. ${ }^{6}$ Due to this effect, we cannot clearly separate the contributions of both subsystems to the GMR. Fe/spacer/ $\mathrm{Fe}(001)$ trilayers with interlayer exchange coupling usually remagnetize via a spin-flop state with the magnetizations along the second easy axis. ${ }^{13}$ Thus, the resistance drop of $\Delta R=5.5 \mathrm{~m} \Omega$ is due to an angle of about $90^{\circ}$ between the topmost $\mathrm{Fe}(2)$ and the central $\mathrm{Fe}(10)$ layer. The saturation field of the structured sample is $190 \mathrm{mT}$, which is more than twice the saturation field of the extended layers (Fig. 1). This dramatic increase can be explained by the competition between the interlayer exchange coupling and external and dipolar fields. The overall GMR ratio defined as $\left(R_{\mathrm{AP}}\right.$ $\left.-R_{\mathrm{P}}\right) / R_{\mathrm{P}}$, where $R_{\mathrm{AP}}$ is the highest resistance value in the fully AP configuration and $R_{\mathrm{P}}$ denotes the smallest resistance in the saturated state, amounts to $2.6 \%$ at RT and $5.6 \%$ at $4 \mathrm{~K}$.

In Fig. 3, positive current corresponds to an electron flow from the top $\mathrm{Fe}(2)$ to the bottom $\mathrm{Fe}(14)$ layer. We observe a parabolic background, which is usually explained by Joule heating. ${ }^{6-9}$ On top of that, we measure field dependent resistance changes, which can be attributed to spin-torque effects. For instance at $-20 \mathrm{mT}, d U / d I$ drops at $I_{C}^{+}=$ $+18.2 \mathrm{~mA}$ from the high-resistivity to a low-resistivity state. After reducing the current again, $d U / d I$ jumps back to the large value. But also at negative bias $d U / d I$ changes at $I_{C}^{-}$ $=-12.1 \mathrm{~mA}$ from large to small. With an estimated junction radius of $a=75 \mathrm{~nm}$ the corresponding critical current densities are $1 \times 10^{8}$ and $-0.7 \times 10^{8} \mathrm{~A} / \mathrm{cm}^{2}$.

The occurrence of jumps at both polarities of the current in small fields is at first glance surprising but can be explained by taking into account that both $\mathrm{Fe} / \mathrm{Cr}$ and $\mathrm{Fe} / \mathrm{Ag}$ subsystems contribute and have spin scattering asymmetries with opposite signs. ${ }^{10,18}$ This leads for the Fe/Cr subsystem to inverse current-induced magnetization switching (CIMS), and the spin torques for the two subsystems are inverted. A negative (positive) current stabilizes the $\mathrm{P}$ (AP) state for 
$\mathrm{Fe} / \mathrm{Ag} / \mathrm{Fe}$ and the $\mathrm{AP}(\mathrm{P})$ state for $\mathrm{Fe} / \mathrm{Cr} / \mathrm{Fe}$. At positive currents, the spin-transfer torque generated in the $\mathrm{Fe} / \mathrm{Cr}$ subsystem destabilizes its AP alignment and switches the $\mathrm{Fe}(10)$ layer (Fig. 3). At negative currents, the $\mathrm{Fe}(2)$ layer gets unstable by the torque created from the $\mathrm{Fe} / \mathrm{Ag}$ subsystem, while the $\mathrm{Fe} / \mathrm{Cr}$ subsystem is stabilized even stronger in the AP state. The dc resistance change measured at $+8 \mathrm{mT}$ (inset of Fig. 3) corresponds to about twice the resistance drop in the inset of Fig. 2 and indicates a complete reversal of $\mathrm{Fe}(2)$ for negative currents (normal CIMS) and $\mathrm{Fe}(10)$ for positive currents (inverse CIMS).

The resistance jumps appear at slightly smaller absolute current values when the external magnetic field $B_{\text {ext }}$ increased by a few $\mathrm{mT}$. This is due to the fact that the magnetic ground state is not stable against an increase of $B_{\text {ext }}$, because the central $\mathrm{Fe}(10)$ layer opposes for small fields the direction of $B_{\text {ext }}$ (Fig. 1). When the field increases, the $\mathrm{Fe}(10)$ layer reverses more easily under the influence of spin transfer, and the positive critical current decreases with increasing $B_{\text {ext }}$. This is opposite to the usual behavior. The sensitivity of the magnetic ground state to $B_{\text {ext }}$ is also important for the $\mathrm{Fe}(2)$ layer, which is aligned with $B_{\text {ext }}$. When $B_{\text {ext }}$ increases, the $\mathrm{Fe}(2)$ layer gets more stable, but the magnetization of the central $\mathrm{Fe}(10)$ layer, which polarizes the current, rotates away from being antiparallel to $B_{\text {ext }}$, and the spin-transfer efficiency $\epsilon(\theta)$ increases. Thus, the negative critical current for the reversal of the topmost $\mathrm{Fe}(2)$ layer decreases with the increasing magnetic field, too. Slonczewski gives the critical current in a pillar ${ }^{19}$ as

$$
\begin{aligned}
& I_{\mathrm{c}}=\frac{e \pi}{\hbar \epsilon(\theta)} a^{2} t \alpha M_{S} B_{\mathrm{eff}}, \\
& \epsilon(\theta)=\frac{\operatorname{sign}(P)}{(1+|P|)^{3}(3+\cos (\theta)) / 4 \mid P^{3 / 2}-4},
\end{aligned}
$$

where the effective field $B_{\text {eff }}$ comprises contributions from the magnetocrystalline anisotropy, the interlayer coupling, and the demagnetizing field. We estimate the titling angle $\Delta \theta$, which is required to compensate the increase in Zeeman energy when $B_{\text {ext }}$ is increased by $\Delta B_{\text {ext }}$, from the condition

$$
\frac{d I_{\mathrm{c}}}{d \theta} \Delta \theta+\frac{d I_{\mathrm{c}}}{d B_{\mathrm{ext}}} \Delta B_{\mathrm{ext}}=0
$$

We find for our experimental conditions and material parameters that a tilting angle $\Delta \theta$ of a few degrees is sufficient to compensate field changes of a few $\mathrm{mT}$, if the initial angle $\theta$ deviates more than $5^{\circ}$ from perfect $\mathrm{P}$ or AP alignment (e.g., $\Delta \theta<2^{\circ}$ for $\Delta B_{\text {ext }}=1 \mathrm{mT}$ and $\theta=5^{\circ}$ ). The latter condition is at least locally fulfilled due to the inhomogeneous magnetization pattern caused by the Oersted field and the edges of the nanopillar. Thus in the low field regime, slight fieldinduced changes of $\theta$ can easily lead to the observed reduction of the switching current for increasing $B_{\text {ext }}$. However, we point out that the switching of the layers at both current polarities cannot be an effect of the Oersted field alone, because the resistance curves are not truly symmetric with respect to the current direction, e.g., the onsets of the resistance drop are -8 and $+17 \mathrm{~mA}$, respectively.

At large magnetic fields exceeding the saturation field, the two thick bottom layers $[\mathrm{Fe}(14)$ and $\mathrm{Fe}(10)]$ are stabilized stronger by the Zeeman energy than the Fe(2) layer, and therefore only one steplike resistance change due to magnetic excitations of $\mathrm{Fe}(2)$ at $I>0$ is observed under these conditions. ${ }^{20}$ For increasing $B_{\text {ext }}$, the steps (i) shift towards larger currents with a rate of $7 \mu \mathrm{A} / \mathrm{mT}$ (dashed line in Fig. 3 ) and (ii) are more pronounced. Both observations are incompatible with magnetization processes induced by the Oersted field but can be explained by (i) the competition between the external field and the spin-transfer torque and (ii) a more homogeneous magnetization and thus spintransfer torque distribution. We use again Eq. (1) to calculate the ratio of the critical currents for the $\mathrm{Fe} / \mathrm{Ag}$ and $\mathrm{Fe} / \mathrm{Cr}$ subsystems and obtain $I_{c}^{\text {th }}(\mathrm{Fe} / \mathrm{Cr}) / I_{c}^{\text {th }}(\mathrm{Fe} / \mathrm{Ag}) \approx-7$. Therefore, the peak corresponding to the excitation of the $\mathrm{Fe}(10)$ layer by the inverse spin-transfer torque should show up at $\approx-7 I_{c}(1166 \mathrm{mT}) \approx-110 \mathrm{~mA}$, which is outside the current range our samples can withstand. Thus, we can only observe the peak at $I>0$ due to the normal spin-transfer torque in the $\mathrm{Fe} / \mathrm{Ag}$ subsystem.

In conclusion, we have prepared single-crystalline nanopillars by MBE and a combined process of optical and e-beam lithographies and observe clear evidence of spintorque effects at current densities of about $10^{8} \mathrm{~A} / \mathrm{cm}^{2}$. This value is mostly determined by the sizable dipolar coupling in the nanopillars. Our pillars contain two subsystems $(\mathrm{Fe} / \mathrm{Cr} / \mathrm{Fe}$ and $\mathrm{Fe} / \mathrm{Ag} / \mathrm{Fe})$ in series, which both give rise to spin-transfer torque effects. The opposite spin scattering asymmetries of $\mathrm{Fe} / \mathrm{Cr}$ and $\mathrm{Fe} / \mathrm{Ag}$ lead in small magnetic fields to the simultaneous occurrence of normal and inverse CIMS in a single nanopillar device. The switching at opposite current polarities provides opportunities for optimizing the CIMS behavior and realizing further magnetic excitation dynamics, e.g., by exciting one subsystem at higher current density while simultaneously suppressing excitations of the fixed layer with the torque exerted by the second subsystem.

${ }^{1}$ M. N. Baibich, J. M. Broto, A. Fert, F. Nguyen van Dau, P. Etienne, G. Creuzet, A. Friederich, and J. Chazelas, Phys. Rev. Lett. 61, 2472 (1988). ${ }^{2}$ G. Binasch, P. Grünberg, F. Saurenbach, and W. Zinn, Phys. Rev. B 39, 4828 (1989).

${ }^{3}$ J. C. Slonczewski, J. Magn. Magn. Mater. 159, L1 (1996).

${ }^{4}$ L. Berger, Phys. Rev. B 54, 9353 (1996).

${ }^{5}$ M. D. Stiles and A. Zangwill, Phys. Rev. B 66, 014407 (2002).

${ }^{6}$ F. J. Albert, J. A. Katine, and R. A. Buhrman, Appl. Phys. Lett. 77, 3809 (2000).

${ }^{7}$ J. Grollier, V. Cros, A. Hamzic, J. M. George, H. Jaffrés, A. Fert, G. Faini,

J. Ben Youssef, and H. Legall, Appl. Phys. Lett. 78, 3663 (2001).

${ }^{8}$ S. Urazhdin, N. O. Birge, W. P. Pratt, Jr., and J. Bass, Phys. Rev. Lett. 91, 146803 (2003).

${ }^{9}$ I. N. Krivorotov, N. C. Emley, J. C. Sankey, S. I. Kiselev, D. C. Ralph, and R. A. Buhrmann, Science 307, 228 (2005).

${ }^{10}$ M. D. Stiles and D. R. Penn, Phys. Rev. B 61, 3200 (2000).

${ }^{11}$ D. E. Bürgler, C. M. Schmidt, J. A. Wolf, T. M. Schaub, and H. J. Güntherodt, Surf. Sci. 366, 295 (1996).

${ }^{12} \mathrm{M}$. Buchmeier (private communication).

${ }^{13}$ M. Buchmeier, B. K. Kuanr, R. R. Gareev, D. E. Bürgler, and P. Grünberg, Phys. Rev. B 67, 184404 (2003).

${ }^{14} \mathrm{C}$. Kittel, Einführung in die Festkörperphysik, 12th ed. (Oldenbourg, München Wien, 1999), p. 478.

${ }^{15}$ Ferromagnetic Materials, edited by E. P. Wohlfarth (North-Holland, Amsterdam, 1980), Vol. 2, p. 512.

${ }^{16} \mathrm{H}$. Namatsu, T. Yamaguchi, M. Nagase, K. Yamazaki, and K. Kurihara, Microelectron. Eng. 41/42, 331 (1998).

${ }^{17}$ P. G. Glöersen, J. Vac. Sci. Technol. 12, 28 (1975).

${ }^{18}$ M. AlHajDarwish, H. Kurt, S. Urazhdin, A. Fert, R. Loloee, W. P. Pratt, Jr., and J. Bass, Phys. Rev. Lett. 93, 157203 (2004).

${ }^{19}$ J. C. Slonczewski, J. Magn. Magn. Mater. 195, L261 (1999); e-print condmat/0205055.

${ }^{20}$ R. Lehndorff, H. Dassow, D. E. Bürgler, and C. M. Schneider (unpublished). 